\title{
El concepto de religión en Esquilo: reflexión terminológica*
}

\author{
Esteban Calderón Dorda \\ Universidad de Murcia \\ esteban@um.es
}

\section{The Concept of Religion in Aeschylus: Terminological Reflection}

Es cosa sabida que los griegos no tenían en su lengua un término equivalente al latín religio, de manera que hicieron uso de una variada gama de términos para expresar esa relación con la divinidad. Esquilo, que pasa por ser un autor que plasma en sus dramas un profundo pensamiento religioso y que a nivel teológico sus planteamientos son de los más relevantes de su época, puede ser un buen laboratorio en el que analizar minuciosamente la diferente terminología que utiliza para definir esa relación con el dios. En este sentido, el término $\varepsilon v ̉ \sigma \varepsilon ́ \beta \varepsilon 1 \alpha$ parece definir mejor que ningún otro esa relación con la divinidad.

Palabras clave: Esquilo; Religión griega; Lexicología.
It is a well known fact that the Greeks had no one term equivalent to the Latin term religio in their language, and therefore made use of a variety of terms to express this relationship with divinity. Aeschylus who is an author who reflected in his plays a deep religious thought and, on a theological level, ideas which are among the most important of his time, could be a good laboratory in which to profoundly analyse the different terminology used to define that relationship with God. In this sense,

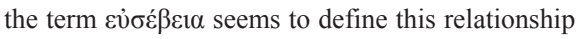
with divinity better than any other term.

Key words: Aeschylus; Greek Religion; Lexico$\operatorname{logy}$.

$\S 1$. El concepto de religión, según nuestra concepción moderna, está indeleblemente marcado por unos condicionamientos morales. Los griegos no tenían ningún libro sagrado que delimitase esa moral, pero a través de los autores literarios podemos intuir cuál era el fuste de esas exigencias que

* Este trabajo ha sido realizado en el marco del Proyecto de Investigación FFI-201126405: Estudio sobre el vocabulario religioso griego. Agradecemos a los informantes anónimos de este trabajo sus sugerencias, que han sido de gran utilidad. Para el texto de Esquilo seguimos la edición de West 1990. 
afectaban a la relación del individuo tanto en su vertiente divina como en su dimensión social. Con todo, siempre existe el riesgo de un desequilibrio que dificulte reconocer en el mundo griego como religiosas realidades o nociones que desde un punto de vista moderno podrían parecer profanas.

Los sentimientos religiosos nos revelan el más sincero comportamiento del ser humano, del pueblo griego en este caso. En un trabajo ya antiguo, Jules Girard $(1879)^{1}$ trató de ahondar en el sentimiento religioso de los griegos, pero con una metodología discutible y escasamente productiva ${ }^{2}$. Lo primero que hay que hacer es distinguir este sentimiento religioso de lo que constituye el dogma o el culto. La religión, según las definiciones tradicionales, se sustancia como un sentimiento de total dependencia de la divinidad ${ }^{3}$. En el caso del autor que vamos a abordar, «el pensamiento religioso de Esquilo sólo podemos conjeturarlo y trazar únicamente algunas líneas generales a través de las siete tragedias que se nos han conservado ${ }^{4}$. Por tanto, se impone un método más preciso y minucioso en el que se excluyan las generalizaciones y las interpretaciones sutiles propias de la subjetividad de cara a conocer cómo es el homo religiosus que nos presenta Esquilo. Para ello conviene recurrir a un análisis más objetivo y de mayor exactitud como es el léxico; dicho de otra manera, parece imponerse un método semántico, aunque no es fácil que las fuentes - literarias en este caso - se pronuncien sobre la naturaleza del sentimiento religioso. Por otra parte, la teología de Esquilo es probablemente la más profunda de su época, lo que nos ayudará en este acercamiento ${ }^{5}$.

§2. Resulta algo normal en las culturas antiguas que el hombre adopte una actitud de distanciamiento temeroso ante aquello que le inspira respeto ( $\sigma \varepsilon \hat{\beta} \alpha \varsigma)$, a la par que lo concibe como algo excelso y sagrado. En el juicio

1 Para el caso de Esquilo contamos también con el trabajo, ya antiguo y muy parcial, de Couffignal 1947, así como la monografía de Kiefner 1965, con un planteamiento muy distinto del que aquí hacemos, ya que, a pesar del título, no se analizan los términos que aquí vamos a estudiar. Más recientemente ha abordado la cuestión, de manera lexicalmente muy parcial y limitada a las Suplicantes y a las Euménides, Bruit Zaidman 2001; las páginas dedicadas a Esquilo y al campo de la $\varepsilon v ̉ \sigma \varepsilon ́ \beta \varepsilon ı \alpha$ realmente quedan reducidas a las pp. 113-118, y son de interés las consagradas al epílogo (pp. 211-217).

${ }^{2}$ Couffignal 1947, p. 328 ss.

${ }^{3}$ Cf. Burkert 2009, p. 145 s.

${ }^{4}$ García López 1975, p. 195 s. Sobre Esquilo puede verse, en líneas generales y con un enfoque alejado de nuestro propósito, Sourvinou-Inwood 2003, pp. 201-263.

5 Sobre este aspecto sigue siendo de interés la lectura del ya clásico libro de Reinhardt 1949. También Wartelle 1965. 


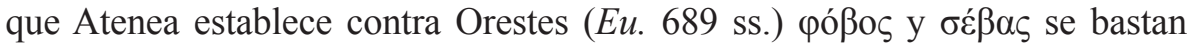
para que el hombre se abstenga de cometer injusticia ${ }^{6}$. Este distanciamiento temeroso es el que se observa en un grupo de palabras relacionadas con el sus-

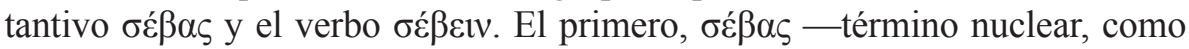
veremos-, representa el temor religioso que inspiran los dioses (Supp. 85;

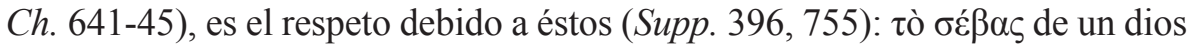
es sagrado, $\dot{\alpha} \gamma$ vó $(E u$. 885). Consecuencia de ello es que el que respeta a los

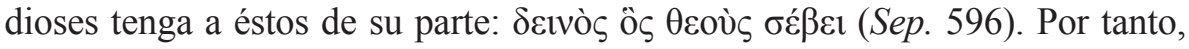

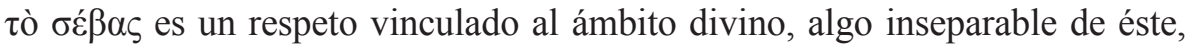
aunque no de manera exclusiva ${ }^{7}$. Es más, en $C h .645$ se trata de un aspecto de

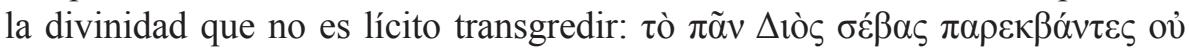
$\theta \varepsilon \mu 1 \sigma \tau \tilde{\omega} \varsigma^{8}$. Dentro del marco tradicional de lo que es la religión, ésta es aceptada como un sistema de rangos, lo que implica dependencia, subordinación; y la divinidad representa poder y, por lo tanto, exigencia de veneración ${ }^{9}$, como la que reciben las Euménides bajo la forma de $\pi \varepsilon \rho i ́ \sigma \varepsilon \pi \tau \alpha$ — por primera vez en Esquilo- compuesto de $\sigma \varepsilon \pi \tau \tau^{\prime} \varsigma^{10}$ y con $\pi \varepsilon p i ́-$ intensivo: las hijas de la Noche obtendrán la mayor reverencia mediante honores y sacrificios $(E u$. 1037).

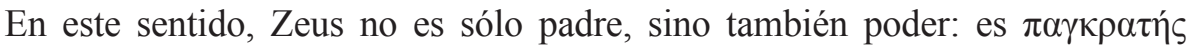
(Sep. 255; Supp. 815; Eu. 918). Sobre esto volveremos. Por eso, la arrogancia

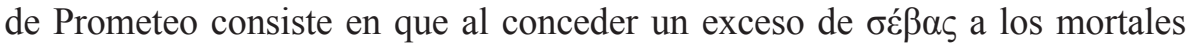
( $P V$ 543), los está acercando a la condición divina y supone una inversión del orden natural ${ }^{11}$; este sentimiento amistoso hacia los hombres ( $\left.\varphi \imath \lambda \alpha v \theta \rho \omega \pi i \alpha\right)$ constituye una suerte de $\dot{\alpha} \mu \alpha \rho \tau i ́ \alpha$ que supondrá su perdición ${ }^{12}$. En relación con este pasaje del Prometeo se ha puesto un verso de un fragmento de la Niobe:

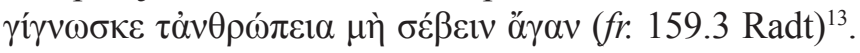

${ }^{6}$ Cf. Jäkel 1975. También resulta interesante otro trabajo del mismo autor (Jäkel 1973), donde analiza los conceptos de timor y pietas a propósito de un caso similar en la Heroida XIV. Sobre este último asunto, cf. Lucas 2008, p. 252 ss.

${ }^{7}$ Cf. fr. 135.1 Radt. Vid. Lucas 2008, p. 443 s.

${ }^{8}$ Hay que señalar, con todo, que los vv. 641-45 ofrecen una lectio incerta que favorece diversas interpretaciones, cf. Garvie 1986, p. 220 s. No obstante, hallamos una expresión

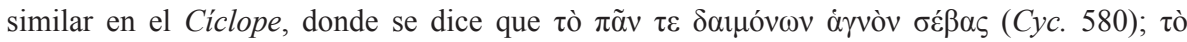

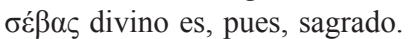

${ }_{9}$ Cf. Burkert 2009, p. 146 ss.; Goward 2005, p. 71.

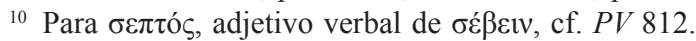

${ }^{11}$ Griffith 1983, p. 186.

12 Cf. Papadopoulou-Belmehdi 2003, p. 55.

${ }^{13}$ Cf. Pennesi 2008, p. 87 s. 


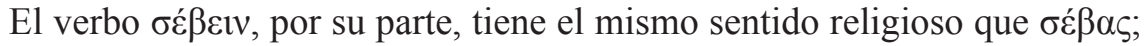
incluso a menudo hace mención expresa de la divinidad ${ }^{14}$. Se utiliza, por

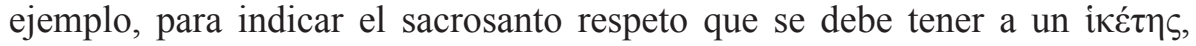

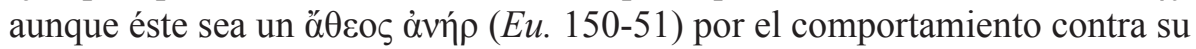

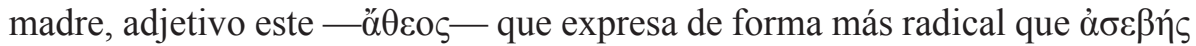
la supresión de la relación con los dioses ${ }^{15}$. Ya Eurípides previene de la situación ciertamente paradójica de Orestes si no ejecuta la venganza por el asesinato de su padre, quedará mancillado, pero si la lleva a cabo y mata a su

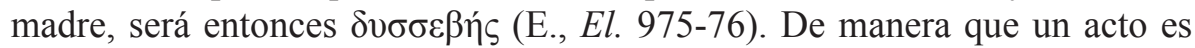
piadoso cuando agrada a los dioses $(C h .637)^{16}$.

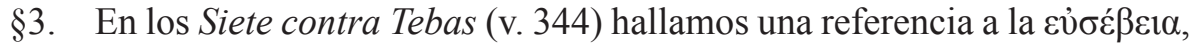
cualidad del $\varepsilon \dot{\sigma \varepsilon \varepsilon \beta \eta ́ \varsigma, ~ q u e ~ p a r e c e ~ r e s p o n d e r, ~ e n ~ n o ~ p e q u e n ̃ a ~ m e d i d a, ~ a ~ u n a ~}$ buena parte de lo que es la religión. El mismo prefijo ev̉- está indicando una cualidad positiva. En dicho pasaje afirma Esquilo que cuando Ares - por metonimia, un ejército - entra a saco en una ciudad, nada es respetado, incluso la $\varepsilon \dot{\sigma} \sigma \dot{\beta} \beta \varepsilon 1 \alpha$, esto es, son destruidos o profanados los lugares sagrados,

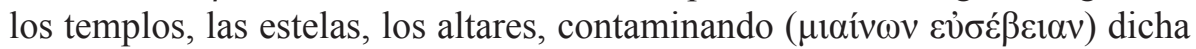

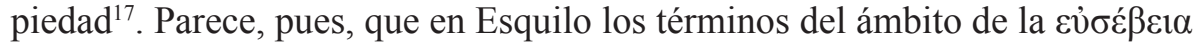
se imponen a la hora de traducir el sentimiento religioso más puro, el núcleo de lo que es la verdadera piedad y, por lo tanto, ocupan una posición de privilegio a la hora de expresar la piedad humana. La $\varepsilon \dot{\sigma} \delta \varepsilon \varepsilon \varepsilon 1 \alpha$ añade un elemento esencial en el comportamiento humano, como es el reconocimiento de su subordinación a la voluntad divina, que impele a respetar la condición humana de los demás individuos ${ }^{18}$.

${ }^{14}$ Zeus (Supp. 671), Atenea (Eu. 435), Ninfas (Eu. 22), el poder celeste (Ch. 960), Apolo (Eu. 12: $\sigma \varepsilon \beta i ́ \zeta \varepsilon v v)$. El adjetivo correspondiente a $\sigma \varepsilon \dot{\beta} \beta \alpha \varsigma, \sigma \varepsilon \mu v o ́ \varsigma(<* \sigma \varepsilon \beta-v o ́ \varsigma)$, puede hacer alusión también a la majestad sagrada de los reyes (Ch. 357; cf. Ch. 975). Vid. Cheyns 1990.

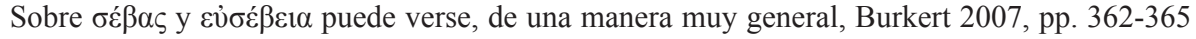
(para őøı̊ y algún otro término abordado más adelante, v. pp. 357-360).

${ }^{15}$ El testimonio más antiguo de este neologismo está en Esquilo, Per. 808.

16 También indica el respeto a los padres (PV 1091) o a un país (Supp. 776-77).

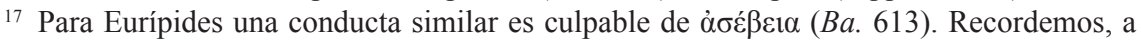
propósito de actos semejantes, la truculenta descripción de Tucídides II 52, y la inversión de valores morales y religiosos de II 53.

${ }_{18}$ Cf. Helm, 2004. También es interesante, con carácter general, el trabajo de Rudhardt 1976. 


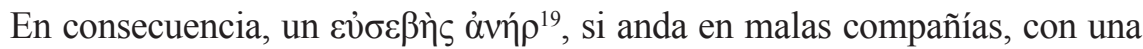
ralea $\theta \varepsilon$ $\pi \tau \tau \sigma \tau o \varsigma$ «detestada por los dioses» (Sep. 604) - hapax —, corre el riesgo de perecer con ella; lo mismo si se trata de personas que resultan enemigas de los huéspedes ( $\left.\dot{\chi} \chi \rho \rho{ }^{\prime} \xi \varepsilon v o l\right)^{20}$ y olvidadizos de los dioses (Sep. 606). Ser $\dot{\varepsilon} \chi \theta \rho o ́ \xi \varepsilon v o \varsigma$ es un crimen particularmente grave, toda vez que supone un rechazo a las leyes de la hospitalidad, cuya observancia está impuesta por Zeus $\xi \dot{v} v 10 \varsigma$ y que tiene una posición significativa en la ética y la teología de los griegos $^{21}$. En el segundo estásimo del Prometeo (vv. 526-60) ) $^{22}$ las Oceánides expresan el deseo de no tener como enemigo a Zeus y de poder observar siempre los deberes rituales y las obligaciones morales hacia los dioses, como vía para llevar una vida plena de esperanza y de alegría. Un ejemplo es el adivino

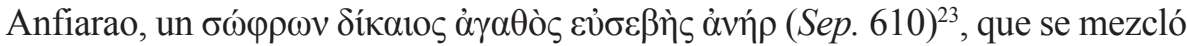
con hombres àvó $\sigma t_{0}{ }^{24}$ (Sep. 611) y se vio arrastrado por ellos, quedando así a expensas de que Zeus le castigue junto con aquéllos. Lo primero que podemos observar al introducirnos en la obra esquílea es que es tanta la preponderancia de Zeus sobre el resto de los dioses que casi se puede hablar de un «monoteísmo» esquíleo. Así, en Agamenón $160-62^{25}$ se puede leer:

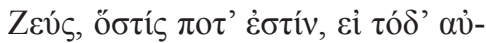

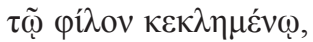

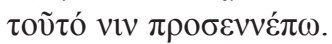

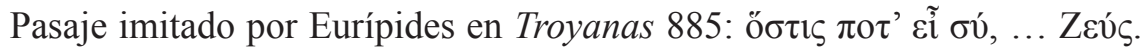
También recuerda las palabras de Melanipa la Sabia (tragedia del 420 a.C.)

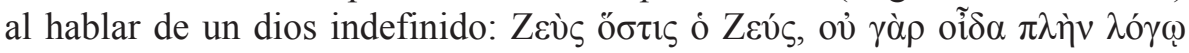

\footnotetext{
${ }_{19}$ Algunos datos de interés sobre éste y otros conceptos pueden verse en el trabajo de Bolkestein 1936, pp. 15-24; pero, como ha señalado Jeanmaire 1945, p. 67: «ce travail ... est plus intéressant, semble-t-il, par la commodité de la reunión d'un assez grand nombre de textes et leur inventaire chronologique que par ses conclusions».

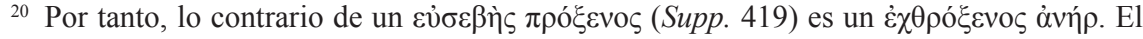

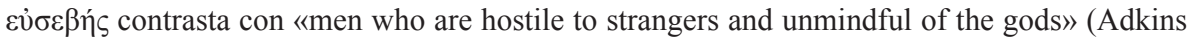

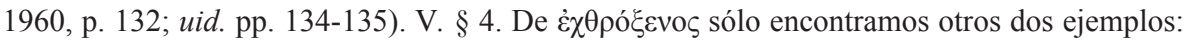

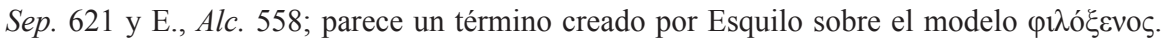

${ }^{21}$ Cf. Lupaş - Petre 1981, p. 195, y Hutchinson 1985, p. 138.

${ }^{22}$ Cf. Griffith 1983, p. 182.

${ }^{23}$ Sobre este catálogo de virtudes, cf. Hutchinson 1985, p. 138.

${ }^{24}$ El adjetivo ávóøioৎ se encuentra por primera vez en Esquilo.

${ }_{25}$ Para la fórmula empleada por Esquilo, uid. Fraenkel 1978, p. 99 s.
} 
(fr. 480 Kannicht), que provocaron el rechazo del público ateniense, según cuenta Plutarco (Amat. 756B) al referirse a este fragmento euripídeo, hasta el punto de que el trágico alteró lo dicho en una segunda versión: Zev́s, ஸ́s

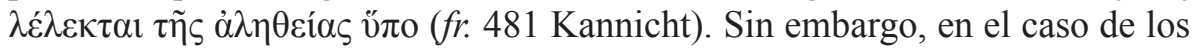
versos del Agamenón, ubicados en su contexto, no ofrecen duda alguna acerca de su piedad o temor reverencial hacia el dios.

En las Helíadas (fr. 70 Radt) también encontramos otra afirmación, según la cual Zeus es el éter, la tierra, el cielo y la totalidad de las $\operatorname{cosas}^{26}$. Esquilo es un eslabón más del proceso que se dio en los siglos VI y V hacia el monoteísmo o henoteísmo ${ }^{27}$ y del que también forman parte Jenófanes, Parménides y Platón, todos ellos adelantados en esta dirección. Esquilo es, como lo ha definido Dodds, «un profeta de la nueva época» ${ }^{28}$. Sin ir en contra del politeísmo tradicional, Esquilo parece encaminarse hacia una nueva «religión de Zeus» ${ }^{29}$.

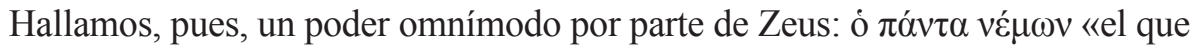

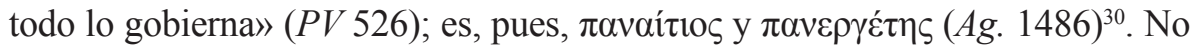
hay que olvidar que, además, en Esquilo ya se observa una tendencia a considerar a Zeus como un instrumento de la justicia ${ }^{31}$. Así es como el hombre es-

${ }^{26}$ Cf. Lucas 2008, p. 316, n. 701. Sobre este pretendido monoteísmo de Esquilo, cf. Deforgue 1986, p. 129, y Scott 1993. En relación con esa especial devoción por un dios concreto que llamamos religión personal, uid. Festugière 1954, y Rudhardt 1976. Sobre la relación entre este fragmento de las Helíadas con Jenófanes, cf. Sommerstein 1996, p. 379. Para la figura de Zeus en el pensamiento esquíleo y su patronazgo sobre la justicia, siguen siendo capitales el artículo de Lloyd-Jones 1965, y su libro: 1971, pp. 78-103, así como el trabajo de Grube 1970.

${ }^{27}$ Cf. Durán 2011, pp. 100-112.

${ }_{28}$ Dodds 1994, p. 60, n. 1.

${ }^{29}$ Cf. Lloyd-Jones 1965, y Parker 2009.

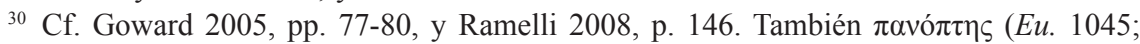

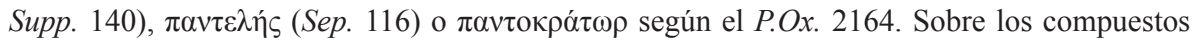
en $\pi \alpha v$ - en relación a Zeus, $v$. la mencionada obra de Kiefner 1965. La ruina (ö $\tau \eta)$ es enviada por Zeus ya en Homero (Il. IX 511-12) y en Solón (fr. 1.75-76 Adr.). En Eu. 199-200 el corifeo aplica a Apolo los adjetivos $\mu \varepsilon \tau \alpha i ́ \tau 1 o \varsigma$ y $\pi \alpha v \alpha i ́ \tau 1 o \varsigma$, que pertenecen al campo forense y que inciden en el hecho de que el dios es corresponsable del homicidio de Orestes (cf. Rossi 1999).

${ }^{31}$ Cf. Dodds 1994, p. 42; Goward 2005, p. 72 s. La Justicia como personificación es hija de Zeus (Sep. 662); ya en Hesíodo, Op. 256. En las Euménides dará un paso más y presentará la institución de la justicia racional a través de Atenea. V. Solmsen 1947. En el fr. 281a Radt se puede ver perfectamente esta vinculación entre Zeus y la Justicia en Esquilo, con la imagen de una tablillas de Zeus (v. 21), en las que la Justicia va registrando las acciones injustas de los hombres (cf. Lucas 2008, p. 683). 
quíleo remite a Zeus sus ansias de justicia. Se podría decir que Zeus encarna el principio de la justicia cósmica ${ }^{32}$. Por tanto, la piedad hacia los huéspedes es un aspecto nuclear en el pensamiento religioso esquíleo. Cuando Pelasgo pre-

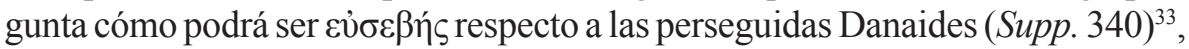
la corifeo responde precisamente que garantizando su asilo al no entregarlas a

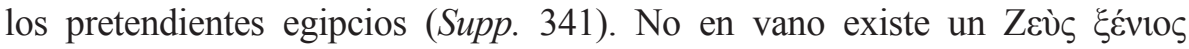
(Supp. 627; cf. Ag. 61, 362, 748; Supp. 672; fr. 451h Radt) ${ }^{34}$. El epíteto de Zeus como $\xi \dot{\varepsilon} v 10 \zeta$, como protector no sólo de los huéspedes, del derecho de hospitalidad, sino también de los extranjeros, lo tenemos testimoniado desde Home-

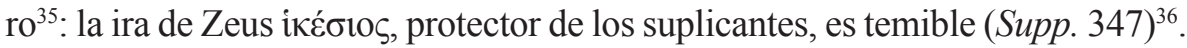
En el caso de $A g .61$ se alude a un tipo de adulterio que viola, a la vez, la santidad del vínculo y la hospitalidad del anfitrión ${ }^{37}$. El respeto $(\sigma \varepsilon ́ \beta \alpha \varsigma)$ a los padres y a los huéspedes debe situarse en primer lugar en la escala de valores del hombre piadoso $(E u$. 545-48). De hecho, el respeto a los progenitores venía,

${ }^{32}$ Cf. Dodds 1994, p. 45 s.; Cohen 1993. En la convulsa época que le tocó vivir a Esquilo se pudo originar la creencia compensatoria de otro tipo de justicia, cf. Ag. 461-67. V. Dodds 1994, p. 55. Piénsese también que ya en el culto Zeus aparece como un cabeza de familia

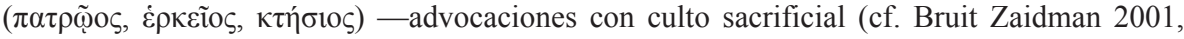
p. 36) - , por lo que es natural proyectar sobre él funciones que corresponden al padre humano; de ahí que en época arcaica aparezca como fuente de dones y, a la par, como juez, justo pero severo, que castiga el pecado de v̋ßpıs, como veremos más adelante (cf. Dodds 1994, p. 57).

${ }^{33}$ En este verso oũv podría sugerir que las Danaides han mencionado algo referente a tò

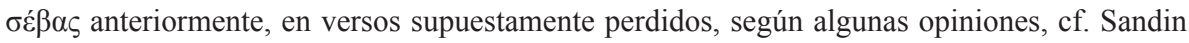
2005, p. 182.

${ }^{34}$ Cf. Winnington-Ingram 1983, p. 63 s. y 68: este autor propone que en las Suplicantes el papel central lo desempeñaba Zeus i $\varepsilon \dot{\sigma} \sigma \mathrm{s}$, mientras que en el desaparecido drama de los

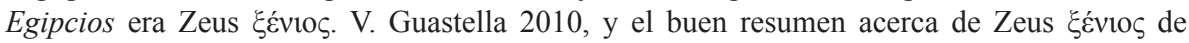
Vürtheim 1967, pp. 6-8. Sobre los problemas textuales del pasaje, cf. Friis Johansen y Whittle 1980, III, pp. 37-39.

${ }^{35}$ Cf. Nilsson 1955, pp. 419-421. En la Odisea (7.164 s.; 9.270 s.; 14.283 s.) Zeus no sólo protege a los suplicantes, sino que hace lo propio con los extranjeros y mendigos (Od. 6.207

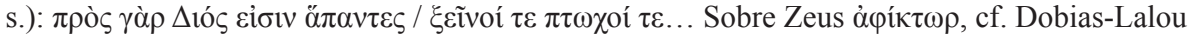
2001. Para la protección de mujeres extranjeras y suplicantes, uid. Santiago, 2005.

${ }_{36}$ Al adornar la imagen de Zeus (Supp. 209-11), las Danaides le han conferido un gran aspecto divino (Sandin 2005, p. 193). Como señala Burkert 2009, p. 149, «la dominación hace posibles formas de solidaridad que no se encuentran fácilmente en otras partes, el precio de aceptar la dependencia de algo que está fuera de nuestro alcance». Ese reconocimiento de superioridad o dependencia de algo superior externo se concreta en las súplicas y sus actitudes, postrándose ante las estatuas de los dioses o abrazándolas (Sep. 95-99).

${ }^{37}$ Fraenkel 1978, p. 39 s. 
en la escala de deberes, inmediatamente después del temor a los dioses; el mismo Esquilo habla del castigo sobrenatural que sufren quienes cometen delitos contra los padres y los huéspedes (Eu. 269-72). Por eso, el que quebranta

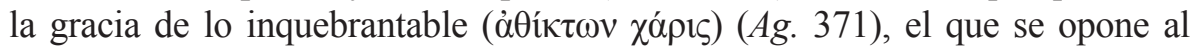
poder de Zeus o se muestra impío, alguien que pisotea lo que es ő $\theta 1 \kappa \tau \alpha^{38}$, ese

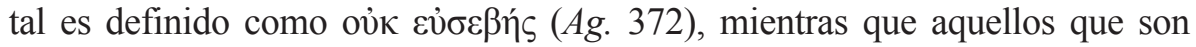
piadosos con los dioses de la ciudad, es decir, los que cumplen con el deber de

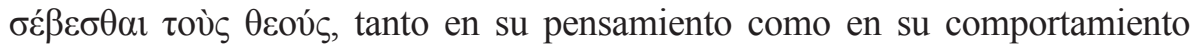
general, no pueden pasar de vencedores a ser vencidos (Ag. 338-40):

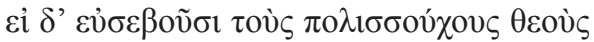

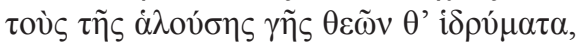

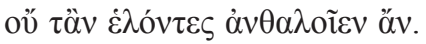

Por el contrario, la $\delta v \sigma \sigma \varepsilon ́ \beta \varepsilon ı \alpha$ es la hospitalidad no correspondida por el huésped (Ch. 704-6) ${ }^{39}$, una impiedad contra los dioses y contra los hombres. Por eso el coro final de las Euménides (1018-19) recuerda a éstas que si

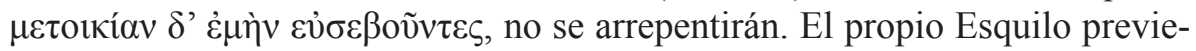

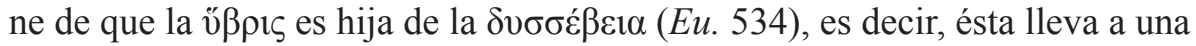
conducta ensoberbecida y desordenada, en la que el humano pierde el norte de su realidad y de su limitación, e infringe aquellas normas elementales que le permiten una relación con la divinidad, a la par que se introduce en una espiral de desgracias ${ }^{40}$ : una obra impía siempre obtendrá en correspondencia un resultado igualmente impío ( $\mathrm{Ag}$. 758-59) ${ }^{41}$ :

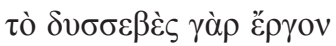

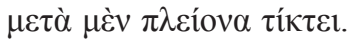

Agamenón probará este amargo café por su decisión de sacrificar a su hija

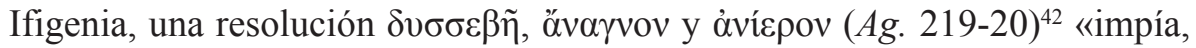

\footnotetext{
${ }^{38}$ Fraenkel 1978, p. 194 s.

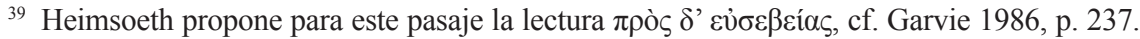

${ }^{40}$ Aquello que se lleva a cabo con v̌ßpıs siempre resulta óvóøıv, según Eurípides $(B a$. 370-75). V. Rudhardt 1958, p. 36 s. y Winnington-Ingram 1983, p. 99. Más recientemente, sobre las consecuencias de la ưßpıs, Ramelli 2008.

${ }^{41}$ Cf. Judet de la Combe 1982, p. 105, n.1.

${ }^{42}$ Como señala Fraenkel 1978, p. 128, estos adjetivos se ajustan a la descripción de algo nefastum.
} 
impura y sacrílega», capaz de concebir un acto abominable, un acto $\pi \alpha v \tau o ́ \tau o \lambda \mu o v ~(A g .221)$, de completa osadía. Es el destino elegido por el

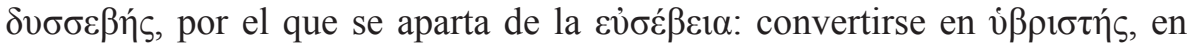
$\pi \alpha v \tau o ́ \tau o \lambda \mu o \varsigma$, esto es, en convertir sus actos en un desafío a la divinidad ${ }^{43}$.

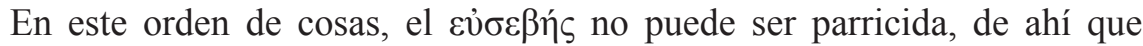
Electra invoque a Agamenón solicitando para ella una mano más piadosa ( $(\dot{\sigma} \sigma \varepsilon \beta \varepsilon \sigma \tau \varepsilon \dot{\varepsilon} \alpha)$ que la de su madre, Clitemestra (Ch. 140-41), es decir, una

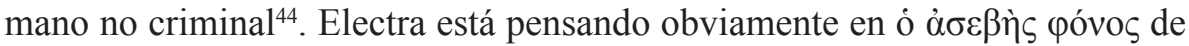
Agamenón. La muerte de Agamenón también constituye un parricidio, un acto $\dot{\alpha} \sigma \varepsilon \beta \eta ́ \varsigma$, , constitutivo de delito impío en el pensamiento religioso griego ( $A g .1493$ y 1517) y la retribución de la impiedad es la ruina. Por esta misma razón Electra, un poco antes, se sorprende de la recomendación del corifeo, cuando éste le sugiere que pida con libaciones en la tumba de su padre el envío de un vengador que dé muerte por muerte, lo que se cumplirá con la llegada de Orestes. La sorprendida Electra pregunta (Ch. 122):

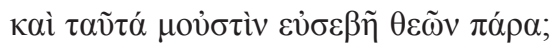

Como se puede observar, el adjetivo indica aquí una disposición del espí-

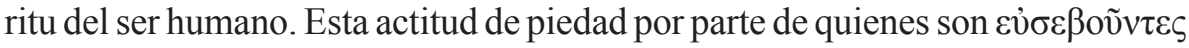
tiene su premio, como lo atestigua el pasaje en el que Atenea solicita a las Euménides una tierra ubérrima y próspera para aquéllos (Eu.910). En el mismo orden de cosas que los casos arriba expuestos, el fratricidio - en el caso

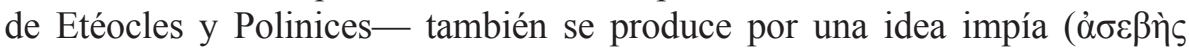
$\left.\delta i^{\prime} v o 1 \alpha\right)$ (Sep. 831), que entraría en la misma categoría de impiedades que las pretendidas bodas de los egipcios con las Danaides: $\alpha \sigma \varepsilon \beta \tilde{\eta} \ldots<\delta$ ióvol $\alpha v>$, en suplemento de Weil (Supp. 10) ${ }^{45}$. Desde el punto de vista de las Danaides, el

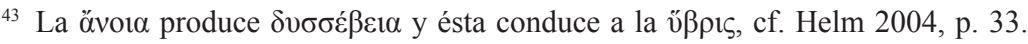

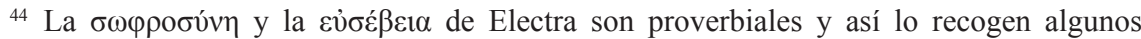
autores como, por ejemplo, Aristófanes, $N u$. 537, donde parece que se alude a la Electra

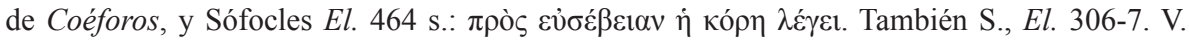
Garvie, 1986, p. 79.

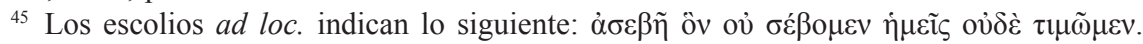
Como Sandin 2005, p. 45 ha señalado, el hecho de que el lema no recoja el final de verso puede indicar que para el escoliasta ya hubiera desaparecido. El suplemento de Weil parece

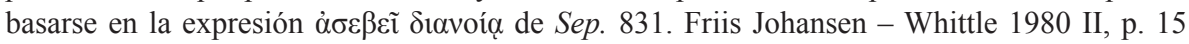
han sugerido que la desaparición de $\delta$ ióvotav pudo producirse a consecuencia de la vecindad del término similar $\Delta \alpha v \alpha o ́ \varsigma$ por parablepsis. 
matrimonio con los hijos de Egipto provoca un doble motivo de indignación: de cara a los dioses es impío, profano y odioso, de cara a los hombres es un delito contra el honor, la dignidad y los derechos legales de las jóvenes ${ }^{46}$. La pretensión de los egipcios viene, además, reforzada por dos características que le confieren un aspecto especialmente desagradable al ejercerla con violencia ( $\beta i ́ \alpha)$ e insolencia (ű $\beta \rho ı \varsigma)$, conceptos netamente esquíleos ${ }^{47}$.

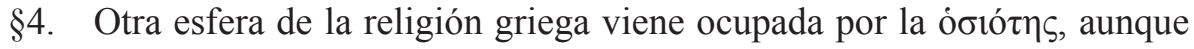
el término como tal no aparezca en Esquilo. Por tanto, el adjetivo öбıৎ designa a aquel que tiene una buena disposición de ánimo hacia la divinidad, al piadoso ${ }^{48}$, así como aquello que es sancionado o permitido por la ley divina o natural ( $P V$ 529). Este adjetivo también designa actos procedentes de ese ánimo como, por ejemplo, los sacrificios ${ }^{49}$ que las Oceánides, aunque diosas, mas de rango inferior a las grandes divinidades olímpicas, ofrecen a los dioses mayores como actos de piedad ( $P V$ 529-31):

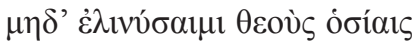

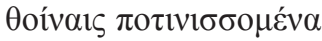

ßovpóvors ...

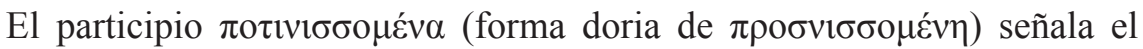
deseo de acercamiento a los dioses, en el que no está ausente tampoco la

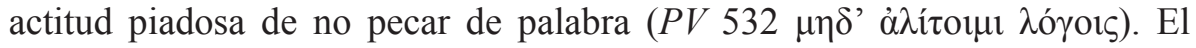
$\dot{\alpha} \lambda ı \tau \omega ́ v$, pues, se aleja del ámbito divino. Esquilo nos ha dejado un claro ejemplo de lo que es ser $\dot{\alpha} \lambda ı \tau \omega ́ v:$ Orestes. Así, en $E u$. 316-17:

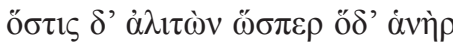

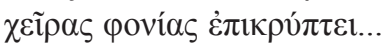

El coro de Erinis da cuenta de uno de los factores que alejan de la divinidad: tener las manos manchadas de sangre por un matricidio, de ahí que persigan a los que tienen $\chi \varepsilon \bar{\rho} \alpha \alpha \varsigma$ povías $(E u$. 317) y a los que tienen unas

${ }^{46}$ Sandin 2005, p. 44 s.

${ }^{47}$ Lucas 2008, p. 168.

${ }^{48}$ Cf. Bolkestein 1936, p. 193.

${ }^{49}$ Los dioses no aceptan los sacrificios más que de los que son ő 43, 65). V. Van der Walk 1941. En la literatura latina también hallamos ejemplos de Ninfas ofreciendo sacrificios: Verg., G. IV 380; Ou., Met. VIII 580; Fast. IV 423. 


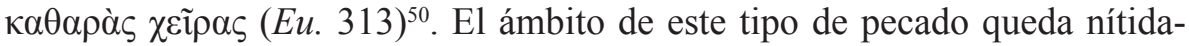
mente delimitado en las Euménides (vv. 269-72):

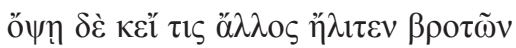

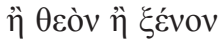

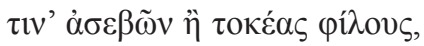

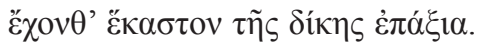

En este pasaje se puede observar que no sólo se peca directamente -Es-

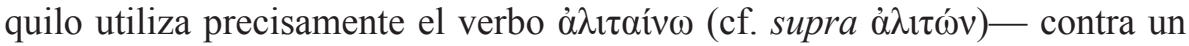
dios, sino también incurriendo en otros aspectos considerados sagrados por el pensamiento religioso griego, como lo son el respeto al huésped y a los progenitores $^{51}$. Las consecuencias son terribles para los que así actúan, ya que Hades lo registra todo en su memoria y ajusta las cuentas bajo tierra $(E u$. 273-75) $)^{52}$. Por tanto, el hombre ő $\sigma ا o \varsigma$ es el que ha cumplido con todas sus obligaciones a ojos de los dioses y, consecuentemente, éstos no tienen nada que reclamarle ${ }^{53}$. Entre estos crímenes execrables se incluye el parricidio, de ahí que las manos de Egisto y de Clitemestra también sean consideradas

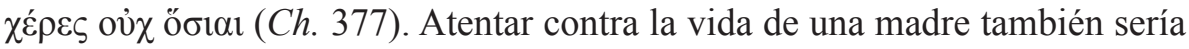
incurrir en una falta contra la aỉós (Ch. 899). El que así actúa es considera-

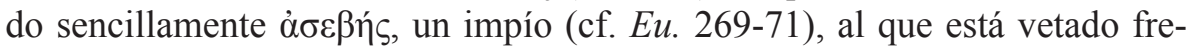
cuentar una comunidad por su mancilla (cf. $E u$. 170) ${ }^{54}$, lo que prueba la dimensión religiosa pero también social de la conducta. No deja de ser elocuente que Orestes sea juzgado por el Areópago al tratarse de un póvo $\pi \rho o v o i ́ \alpha \varsigma$ y no por el Delfíneo, que entendía de los homicidios justificados, de ahí que Apolo, que asume su defensa, trate de presentar el caso como un

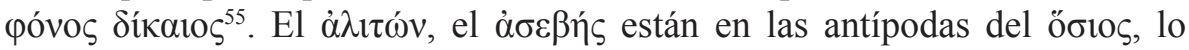
mismo que el $\delta \dot{\sigma} \sigma \varepsilon \varepsilon \varsigma$, que es como nuestro trágico llama a Atreo por su abominable crimen de descuartizar a los hijos de Tiestes $(A g .1590)$ y a Cli-

${ }^{50} \mathrm{La}$ pureza, en la religión griega, no es moral, sino material.

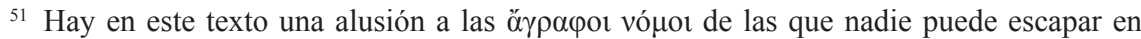
todo el universo: cf. el famoso pasaje de Sófocles, Ant. 453-60. V. Cerri 1979, pp. 33 ss., 53 ss., 65 ss., 79 ss., 102 ss.

52 En Supp. 155-60 Hades es considerado como una hipóstasis de Zeus.

${ }_{53}$ Cf. Van der Walk 1941, p. 118, y Jeanmaire 1945.

${ }^{54}$ De ahí las purificaciones llevadas a cabo por Orestes y de las que nos habla Esquilo en las Euménides 274-86.

${ }^{55}$ Cf. Rossi 1999, p. 204. 


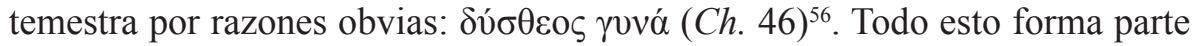

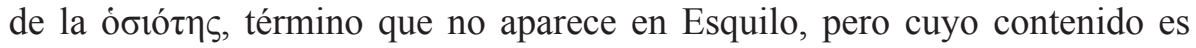
deducible a partir de los datos examinados.

En definitiva, Esquilo resume el comportamiento del ö $\sigma ı \varsigma$ como aquel que vive de acuerdo con las leyes ${ }^{57}$, y no sólo con las leyes religiosas, sino también con los preceptos morales relativos a los hombres, como son la protección del débil y del suplicante ${ }^{58}$, habida cuenta de que la súplica al dios, de manera directa o a través de un lugar sagrado, era una institución religiosa y social muy importante en la Antigüedad y que define el comportamiento humano dentro de un marco divino ${ }^{59}$. Ésta es la actitud de Pelasgo en las

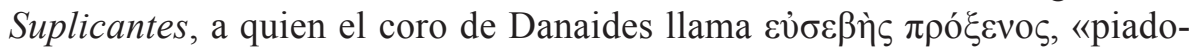
so anfitrión» (Supp. 419), incidiendo en esa faceta que ya hemos visto que caracteriza al ő $\sigma ı \varsigma$ y que consiste en considerar sagrados a los huéspedes

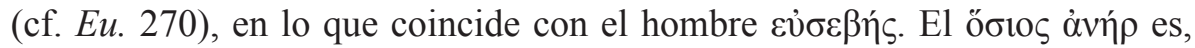
así, visto con agrado y con buenos ojos por Zeus, quien se convierte en su

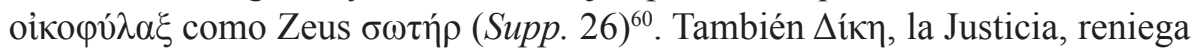
de las manos manchadas por la impiedad y prefiere habitar aquellas casas que son ö $\sigma 1 \alpha$ ( $A g$. 776-81), que siguen un culto adecuado y donde no residen

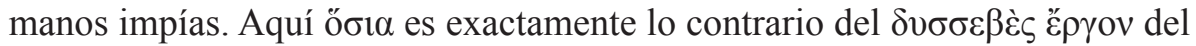
v. 758, las casas ö $\sigma ı \alpha$ son el resultado de las acciones particulares que hacen

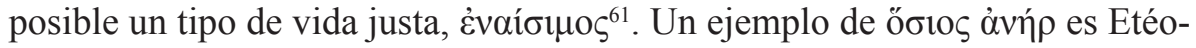
cles, que obtuvo una sepultura digna precisamente por haber respetado $\tau \grave{\alpha}$ iєра̀ $\pi \alpha \tau \rho \tilde{\omega} \alpha$ (Sep. 1010; cf. S., Ant. 194-97), es decir, todos aquellos ritos ancestrales que conformaban la religión patria, frente a Polinices, que quedó insepulto por haber actuado contra los dioses patrios (Sep. 1018).

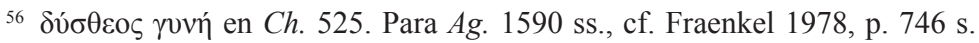

57 Cf. Supp. 402-4. Aunque lo que Zeus tiene más en cuenta es la distinción entre el bien y el mal (uid. Sandin 2005, p. 195 s.).

${ }^{58}$ V. Hall Strenberg 2005. No en vano hallamos por vez primera en Teognis (132) la ex-

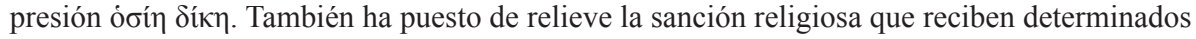
deberes cívicos, como los ya señalados, Jouan, 1979, p. 367. Friis Johansen - Whittle 1980, II, p. 321: «Putting the ö $\delta ı \kappa \alpha$ of the wicked on their scale and the ö $\sigma 1 \alpha$ of the law-abiding on theirs».

${ }_{59}$ Papadopoulou 2011, p. 39. Cf. Gould 1973.

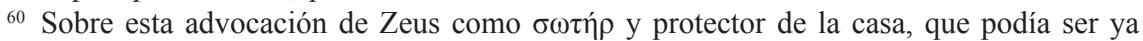
tradicional en tiempos de Esquilo, y el brindis en las libaciones, cf. Friis Johansen - Whittle 1980, II, p. 27, y Sandin 2005, p. 55 s. V. fr. 55 Radt.

${ }^{61}$ Cf. Judet de la Combe 1982, p. 133 ss.
} 
Como consecuencia de todo lo anterior, Esquilo recomienda no mezclarse

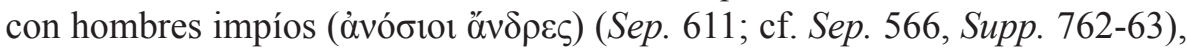
ya que las conductas impías acaban en una muerte miserable (Sep. 550-51).

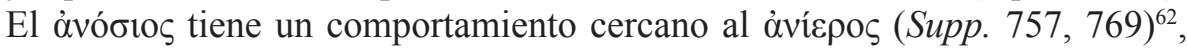

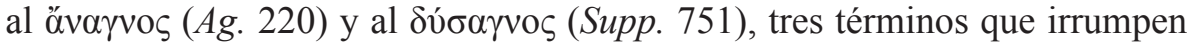
negativamente en el campo semántico de lo sagrado ${ }^{63}$. Algo parecido ocurre

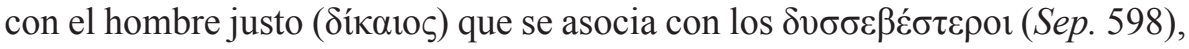

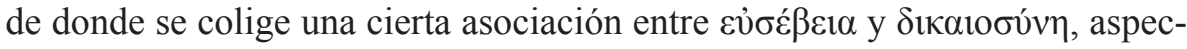
to en el que parece coincidir con la óøió $\rceil$ s. La verdadera piedad está asociada a la justicia y a una serie de vónor (cf. Eu. 539-47; Supp. 698-709) ${ }^{64}$;

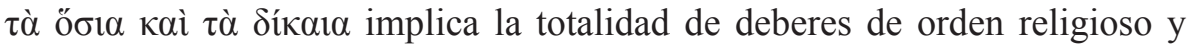
social (cf. Pl., R. 301D; Lg. 663D), son las prácticas y usos permitidos o prohibidos en una comunidad, aquello que está establecido como regla en la conducta humana para con los dioses y para con los hombres. Estamos, pues, ante el viejo problema de la existencia del mal en el mundo: el malvado no puede recibir de parte de los dioses la misma retribución que el justo, lo que nos lleva a una consideración moral de la conducta del individuo piadoso y justo. En este sentido, se considera moralmente lógico que el malvado vea el castigo en su descendencia: es el mensaje de la Orestía ${ }^{65}$. El hombre ő $\sigma 10 \varsigma$ es, pues, aquel que tiene una buena disposición de ánimo hacia la divinidad y realiza acciones que están al servicio de la misma. Se podría decir que este adjetivo encarna una calidad humana. La óøió $\rceil \zeta$, pues, se definiría en relación a un orden objetivo, en el cual el individuo está en armonía con los demás, puesto que su campo marca los límites de lo que es i $\varepsilon \rho o ́ \varsigma^{66}$, mientras

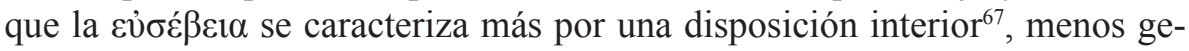
neral que la anterior.

${ }^{62}$ Cf. $A g .768$ s., Judet de la Combe 1982, p. 119 ss.

${ }^{63}$ Son ővorva, por ejemplo, los actos criminales de Clitemestra (Ch. 986).

${ }^{64}$ Cf. Van der Walk 1941, p. 134.

${ }_{65} \mathrm{Tal}$ y como señala Dodds 1994, p. 44, la idea de la culpa heredada y el castigo diferido cobra sentido en una sociedad en que la familia es concebida como una unidad moral; nada más lógico, pues, que los hijos hereden las deudas morales de los progenitores como se heredan las deudas comerciales. Todavía Platón, en el s. IV a.C., cuenta que algunos in-

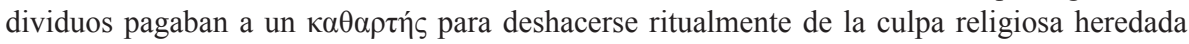
(Tht. 173D; R. 364B-C).

${ }_{66}$ Burkert 2007, p. 359.

${ }^{67}$ Cf. Rudhardt 1958, p. 15. 


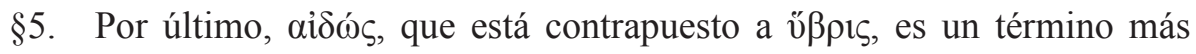
vago $^{68}$, aunque admite una acepción religiosa: describe una actitud de respeto mezclado con temor hacia lo sagrado, un temor reverencial. Es la forma de temor ante aquello que se presenta como un poder que exige respeto. En este sentido, la $\alpha i \delta \omega ́ \varsigma$ religiosa implica el respeto a las instituciones sagradas, algo que está en la línea marcada por la $\varepsilon \dot{\sigma} \varepsilon \dot{\beta} \beta \varepsilon 1 \alpha$, aunque se observa

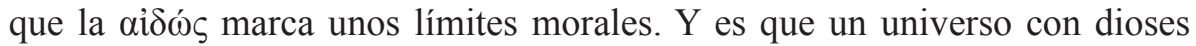
es un universo con normas. Esa vinculación con la $\varepsilon \hat{\sigma} \sigma \varepsilon \beta \varepsilon ı$ se puede comprobar en la actitud de los persas, que actuaron sacrílegamente en la Hélade robando las estatuas de los dioses, incendiando los templos y destruyendo los altares, no respetando así la aỉ $\delta$ ́́ debida a la divinidad en un sentido amplio (Per. 809-12) ${ }^{69}$, ya que, de haberla tenido, habrían reconocido la incorrección de su conducta ${ }^{70}$. Es preciso respetar los altares (Ch. 106) y las tumbas (Ch. 108): son objeto de aỉó́s aquellos sentimientos constitutivos de $\varepsilon \dot{\sigma \varepsilon ́} \beta \varepsilon ı \alpha$. Las suplicantes de un dios también deben ser objeto de respeto sacro (Supp. 641), como partícipes de un respeto que emana del dios: es el

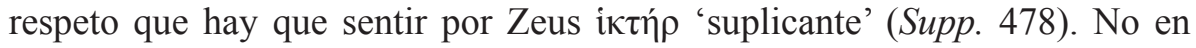
vano las Suplicantes empiezan por estas palabras: Zev̀ $\mu \dot{\varepsilon} v \dot{\alpha} \varphi \hat{i} \kappa \tau \omega \rho$ (v. 1),

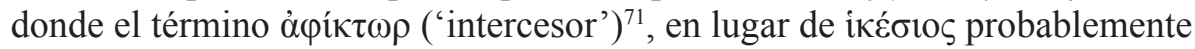
sea una innovación de Esquilo ${ }^{72}$, de suerte que ö $\varphi \alpha \xi 1 \varsigma$, de Supp. 483, sería un sinónimo de i $\kappa \varepsilon \sigma i \alpha^{73}$. Es, pues, un concepto que afecta a un terreno que ya

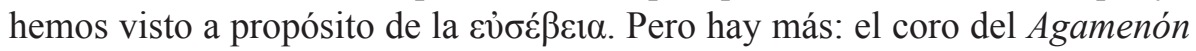

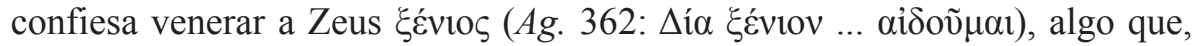
como ya hemos visto arriba, también pertenece al ámbito de la $\varepsilon \dot{\sigma \varepsilon ́} \beta \varepsilon 1 \alpha$. Por lo tanto, se puede considerar que indica igualmente un tipo de ligazón entre el hombre y la esfera divina. En Coéforos 896-99 plantea Esquilo en la persona

${ }^{68}$ Cf. Rudhardt 2001.

${ }^{69}$ Igualmente se respeta un juramento $(E u$. 680, 710). En un fragmento de las Cardado-

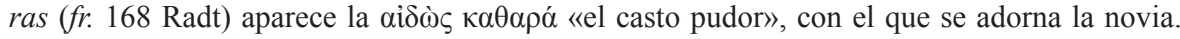

${ }_{70}$ Para el catálogo de actos impíos de los persas, uid. Broadhead 1960, p. 201 s.

${ }^{71}$ Como ha señalado Sourvinou-Inwood (2003, p. 208) en esta expresión «there is an extraordinary density of religious language».

${ }^{72}$ Cf. Supp. 347, 360, 616; uid. también 385, 478 s. y Winnington-Ingram 1983, p. 63 s. V. Vürtheim 1967, p. 163; Friis Johansen y Whittle 1980, II, p. 6; Dobias-Lalou 2001; Sandin 2005, pp. 38-40; Papadopoulou 2011, pp. 39-43. El sufijo - $\tau \omega \rho$ habitualmente designa a los

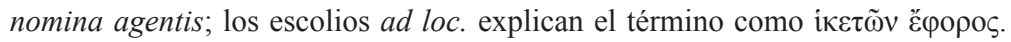

${ }^{73}$ Cf. Vürtheim 1967, p. 163. 
de Orestes una dicotomía religiosa de gran sustancia, que resolverá de la siguiente manera: obedecer a la divinidad está por encima del respeto a la vida de los progenitores. Hay una confrontación de piedades: la que se impone es

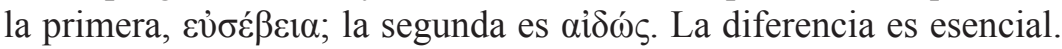

§6. Conclusión. Es evidente que en Esquilo, más allá de las cosas visibles, se extiende otra realidad intangible: $\tau$ à $\theta \varepsilon \tilde{\omega} v$ (Supp. 1061). En su obra queda patente que el hombre no está solo a lo largo de su realidad vital: se percibe una confianza en lo sobrenatural, y los términos empleados por el trágico y aquí estudiados indican la manera de relacionarse con esa esfera sobrenatural, que requiere una actitud piadosa en todo momento. En el caso que nos ocupa, más que de religio o religación, habría que hablar de «relación» con la divinidad, así como de condiciones y actitudes para poder hacerlo. Y aquí hay una diferencia esencial acerca de la esfera religiosa: el hombre griego que refleja Esquilo en sus tragedias accede a esa esfera de lo sagrado cuando los dioses se lo conceden por haber cumplido determinados ritos y obligaciones, como hemos visto. Todo ello queda plasmado a través de su léxico, algunos de cuyos términos aparecen por vez primera en nuestro trágico.

No hay dogmas, hay piedad y hay unos dioses que no son ajenos al universo de los mortales. La religión griega, según parece, no requería una adhesión profunda a un dogma ${ }^{74}$, cosa que, como ya hemos dicho, no existe, sino el simple respeto a los ritos. Sin dogma la noción de piedad es difícil de percibir; por el contrario, la noción de impiedad lo es menos. Por impiedad $(\dot{\alpha} \sigma \varepsilon ́ \beta \varepsilon 1 \alpha)$ los griegos entendían la ausencia de respeto a los diferentes aspectos de la religión, pero especialmente se relaciona con el parricidio, el matricidio o el fratricidio, actos que ciertamente caen fuera de todo $\sigma \varepsilon ́ \beta \alpha \varsigma$, mientras que la $\delta v \sigma \sigma \varepsilon ́ \beta \varepsilon 1 \alpha$ se refiere a actos impíos de menor gravedad, aunque en alguna ocasión puede hacer referencia al delito de sangre: es el caso de Agamenón y su decisión de que Ifigenia sea inmolada, pero, como sabemos, su muerte es evitada en el último instante ${ }^{75}$. Esta es la concepción de $\delta v \sigma \sigma \varepsilon ́ \beta \varepsilon ı \alpha$ en Esquilo; en otros autores - por ejemplo, Eurípides - variará. Por lo tanto, la impiedad tiene poco que ver con la ausencia de fe, sino contra la piedad. Se podría

${ }^{74}$ La religión homérica ya había hecho posible una teología no dogmática.

75 Como ha señalado Judet de la Combe 2009, p. 290 s.: «La mise à mort d'Iphigénie n'est pas pour elle une crime qu'Agamemnon devrait payer, mais un acte de piété, une reconnaissance; c'est une offrande, qui est déjà le prix à payer pour l'entreprise contre Troie». 


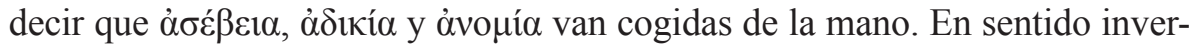
so piedad y justicia también caminan juntas. Se podría definir la piedad griega — piedad (que no fe) real para los griegos - como lo contrario de la impiedad, y aquí nos hallaríamos ante el respeto a las tradiciones y principios ancestrales, el conocimiento de los límites que marcan las leyes divinas y el cumplimiento con las obligaciones que se tiene para con los dioses (ofrendas, plegarias, sacrificios). En este ambiente adquieren pleno sentido los sacrificios, así como las ofrendas, que son tenidas como $\varepsilon \dot{\sigma} \sigma \varepsilon \beta \tilde{\eta}$ (fr. 78a.2-12 Radt ${ }^{76}$.

El respeto a los progenitores, a los huéspedes, a los suplicantes..., refleja un sentimiento religioso idóneo para relacionarse con la divinidad. La piedad designa, pues, la relación con los parientes, amigos y aliados, relación que implica una ayuda leal y fiel. Como dirá Eurípides más adelante ( $P h .525)$, la piedad debe aplicarse a los demás. Dicho de otro modo: la piedad se manifiesta en actos y todo el campo semántico de $\varepsilon v ̉ \sigma \varepsilon ́ \beta \varepsilon \varepsilon \alpha$ ( $\sigma \dot{\varepsilon} \beta \alpha \varsigma$ y sus compuestos y derivados) apunta al respeto como norma fundamental. En este

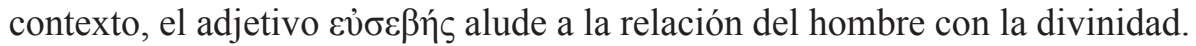
En esa relación el hombre aparece limitado y es entonces cuando deberá encontrar la vía de la purificación para volver a encontrar la óøió $\rceil\rceil s$, para volver a entrar en el camino de lo permitido: el ő $\sigma 10 \varsigma$ es el individuo que está

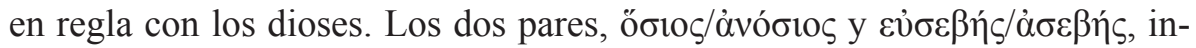
dican unas relaciones en que la religión y la moral no admiten distinción:

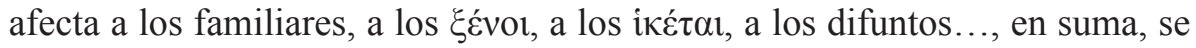
produce en un contexto cívico ${ }^{77}$.

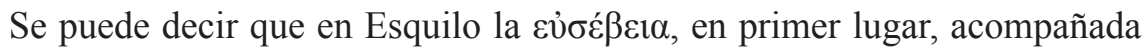

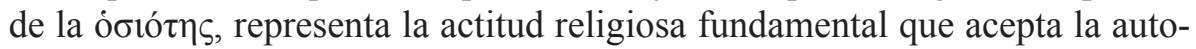
ridad soberana de la divinidad, de Zeus. En este sentido el concepto de aíó́s coadyuva a la definición de $\varepsilon v j \sigma \varepsilon ́ \beta \varepsilon ı \alpha$ insistiendo en su dominio desde una perspectiva más social que religiosa. En definitiva, no se trata sólo de una reducción a la observancia de los actos de culto, sino que consiste fundamentalmente en una conducta personal referida a una serie de ámbitos concretos. La piedad es la fidelidad a los deberes religiosos y estos ejercicios de piedad engloban las relaciones del individuo con otros miembros de la comunidad, actitudes que ayudan a «relacionarse» con la divinidad.

${ }^{76}$ Cf. Lucas 2008, p. 337.

${ }^{77}$ Cf. Nilsson 1953, p. 16: «La religión griega estaba indisolublemente vinculada a la sociedad y a sus elementos constitutivos —polis, genos, familia—». 


\section{BIBLIOGRAFÍA}

Adkins, A.W.H. 1960: Merit and Responsibility: A Study in Greek Values, Oxford.

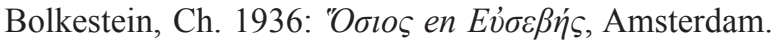

Broadhead, H.D. 1960: The Persae of Aeschylus, Cambridge.

Bruit Zaidman, L. 2001: Le commerce des dieux: eusebeia, essai sur la piété en Grèce ancienne, Paris.

Burkert, W. 2007: Religión griega arcaica y clásica, Madrid, (Stuttgart 1977).

Burkert, W. 2009: La creación de lo sagrado. La huella de la biología en las religiones antiguas, Barcelona (Harvard 1996).

Cerri, G. 1979: Legislazione orale e tragedia greca, Napoli.

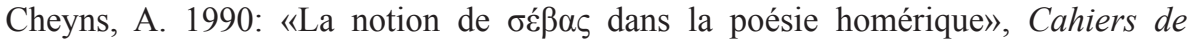
l'Institut de linguistique de Louvain 16, pp. 115-137.

Cohen, D. 1993: "The Theodicy of Aeschylus: Justice and Tyranny in to the Oresteia» en McAuslan, I. y Walcot, P. (eds.), Greek Tragedy, Oxford, pp. 45-57.

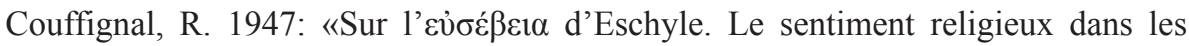
Choéphores étudié d'après quelques vocables», RecSR 34, pp. 328-346.

Deforgue, B. 1986: «Eschyle et la terre divine», en Jouan, F. y Deforgue, B. (eds.), Peuples et pays mythiques, Paris, pp. 123-132.

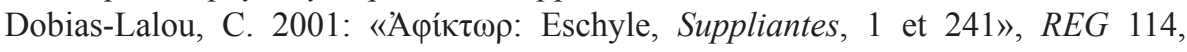
pp. 614-625.

Dodds, E.R. 1994: Los griegos y lo irracional, Madrid (University of California 1951).

Durán López, Ma Á. 2011: Los dioses en crisis. Actitud de los sofistas ante la tendencia religiosa del hombre, Madrid.

Festugière, A.-J. 1954: Personal Religion among the Greeks, Berkeley-Los Angeles.

Fraenkel, E. 1978: Aeschylus. Agamemnon (II-III), Oxford (= 1950).

Friis Johansen, H. y Whittle, E.W. 1980: Aeschylus: The Suppliants (II-III), Copenhagen.

García López, J. 1975: La Religión Griega, Madrid.

Garvie, A.F. 1986: Aeschylus. Choephori, Oxford.

Girard, J. 1879: Le Sentiment religieux en Grèce, Paris.

Gould, J. 1973: «Hieteia», JHS 93, pp. 74-103.

Goward, B. 2005: Aeschylus: Agamemnon, London.

Griffith, M. 1983: Aeschylus. Prometheus Bound, Cambridge.

Grube, G.M.A. 1970: «Zeus in Aeschylus», AJPh 91, pp. 43-51.

Guastella, G. 2010: «Da Zev̀ $\Xi \varepsilon ́ v i o \varsigma$ al fantasma di Tieste: vendetta e giustizia in Eschilo e Seneca», DeM 1, pp. 81-118.

Hall Strenberg, R. 2005: Pity and Power in Ancient Athens, New York.

Helm, J.J. 2004: «Aeschylus' genealogy of morals», TAPhA 134, pp. 23-54. 
Hutchinson, G.O. 1985: Aeschylus. Septem contra Thebas, Oxford.

Jänkel, S. 1973: «The 14th Heroid letter of Ovid and the Danaid trilogy of Aeschylus», Mnemosyne 26, pp. 239-248.

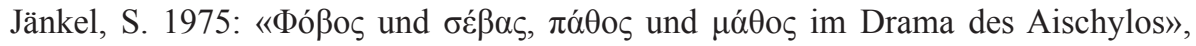
Eirene 13, pp. 43-76.

Jeanmaire, H. 1945: «Le substantif ó $\sigma i ́ \alpha$ et sa signification comme terme technique dans le vocabulaire religieux», REG 58, pp. 66-89.

Jouan, F. 1979: «Rites et croyances: quelques problèmes chez Pindare et Eschyle», $B A G B$ 38, pp. 354-367.

Judet de la Combe, P. 1982: Agamemnon 2. L'Agamemnon d'Eschyle, Lille.

Judet de la Combe, P. 2009: «Les tragedies d'Eschyle sont-elles tragiques?», en Hernández, A.-Ch. (ed.), Eschyle à l'aube du théâtre occidental, Entretiens F. Hardt, t. LV, Vandeoeuvres-Genève, pp. 257-318.

Kiefner, W. 1965: Der religiöse Allbegriff des Aischylos, Hildesheim.

Lloyd-Jones, H. 1965: «Zeus in Aeschylus», JHS 76, pp. 55-67.

Lloyd-Jones, H. 1971: The Justice of Zeus, Berkeley-Los Angeles-London.

Lucas de Dios, J.-Ma 2008: Esquilo. Fragmentos. Testimonios, Madrid.

Lupaş, L. y Petre, Z. 1981: Commentaire aux Sept contre Thèbes d'Eschyle, Bucureşti - Paris.

Nilsson, M.P. 1953: Historia de la religiosidad griega, Madrid (Stockholm 1946).

Nilsson, M.P. 1955: Geschichte der griechischen Religion, vol. I, München (2a ed.).

Papadopoulou, Th. 2011: Aeschylus: Suppliants, Bristol.

Papadopoulou-Belmehdi, I. 2003: "“Les mots qui voient”. Du tragique dans le Prométhée enchaîné», Kernos 16, pp. 43-57.

Parker. R. 2009: «Aeschylus' gods: drama, cult, theology», en Hernández, A.-Ch. (ed.), Eschyle à l'aube du théâtre occidental, Entretiens F. Hardt, t. LV, Vandeoeuvres-Genève, pp. 127-164.

Pennesi, A. 2008: I frammenti della Niobe di Eschilo, Amsterdam.

Ramelli, I. 2008: «Il pensiero teologico ed etico di Eschilo. Nuove note per uno studio filosofico integrato delle tragedia eschilee», Sileno 34, pp. 113-164.

Reinhardt, K. 1949: Aischylos als Regisseur und Theologe, Bern.

Rossi, L.E. 1999: «Strategie oratorie nelle Eumenide di Eschilo», SemRom 2, pp. 199-212.

Rudhardt, J. 1958: Notions fondamentales de la pensée religieuse et actes constitutifs du culte dans la Grèce classique, Genève.

Rudhardt, J. 1976: «Religion sociale et religión personnelle dans la Grèce antique», Dialectica 30, pp. 267-276.

Rudhardt, J. 2001: «Quelques remarques sur la notion d'aidôs», en Delruelle, E.

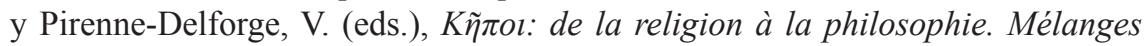
offerts à A. Motte, Liège, pp. 1-21. 
Sandin, P. 2005: Aeschylus' Supplices. Introduction and Commentary on vv. 1-523, Lund.

Santiago Álvarez, R.-A. 2005: «Acogida y protección de mujeres extranjeras: el testimonio de Suplicantes de Esquilo», en Nieto Ibáñez, J.Mª (ed.), La mujer en la cultura griega y latina, León, pp. 143-176.

Scott, S. 1993: «Le Dieu d'Eschyle», RHPhR 73, pp. 249-259.

Solmsen, F. 1947: «Strata of Greek Religion in Aeschylus» HThR 40, pp. 211-226.

Sommerstein, A.H. 1996: Aeschylean Tragedy, Bari.

Sourvinou-Inwood, Chr. 2003: Tragedy and Athenian Religion, Lanham.

Van der Walk, M.H.A.L.H. 1941: «Zum Worte ǒбı૬̧», Mnemosyne 10, pp. 113-140. Vürtheim, J. 1967: Aischylos' Schutzflehende, Groningen.

Wartelle, A. 1965: «La pensée théologique d'Eschyle», BAGB 4, pp. 535-580.

West, M.L. 1990: Aeschylus. Tragoediae, Stuttgart.

Winnington-Ingram, R.P. 1983: Studies in Aeschylus, Cambridge.

Fecha de recepción de la primera versión del artículo: 20/06/2012

Fecha de aceptación: 13/12/2012

Fecha de recepción de la versión definitiva: 11/01/2013 